\title{
Efecto de la intensidad de la luz lunar y de la velocidad del viento en la actividad de murciélagos filostómidos de Mena Nizanda, Oaxaca, México
}

\author{
Effect of the intensity of the moonlight and wind speed in the activity of phyllostomid bats of \\ Mena Nizanda, Oaxaca, Mexico
}

\author{
Antonio Santos-Moreno,*, Elder Ruiz Velásquez² y Abraham Sánchez Martínez² \\ ${ }^{I}$ Laboratorio de Ecología Animal, Centro Interdisciplinario de Investigación para el Desarrollo Integral regional, Unidad Oaxaca, IPN. Hornos 1003, \\ Santa Cruz Xoxocotlán 71230 Oaxaca, México. \\ ${ }^{2}$ Gestión e Investigación para la Conservación y Aprovechamiento de los Recursos Bióticos de Oaxaca, S. C. Almendros 200, Col. Reforma, 68050 \\ Oaxaca de Juárez, Oaxaca, México. \\ *Correspondencia: asantosm90@hotmail.com
}

\begin{abstract}
Resumen. Se analiza la influencia de la velocidad del viento y de la intensidad de la luz lunar en la actividad de 4 especies de murciélagos filostómidos de la región Mena Nizanda, Juchitán, Oaxaca. Se encontró que en conjunto existe una relación significativa e inversa entre la actividad y la velocidad del viento, mientras que la intensidad de la luz lunar no mostró asociación estadísticamente significativa con ella. La mayor actividad se observó con vientos de 15.5 a $24 \mathrm{~km} / \mathrm{h}$ y luminosidad alta (4 en una escala de 0 a 5). Al considerar únicamente a Choeriscus godmani, una especie nectarívora de talla pequeña que fue la más abundante de la comunidad, la actividad estuvo correlacionada en forma positiva y significativa con la intensidad de la luz lunar, mientras que con la velocidad del viento la relación fue inversamente proporcional y también significativa. Para 3 especies frugívoras especialistas en Ficus y de talla grande del género Artibeus no se encontró relación significativa entre la actividad y alguna de las 2 variables. Los resultados anteriores, junto con el hecho de que el número de capturas de C. godmani y las especies de Artibeus no muestran correlación significativa, apoyan la hipótesis de que los patrones de actividad de los murciélagos se ven afectados por los 2 factores ambientales estudiados en forma especie-específica.
\end{abstract}

Palabras clave: murciélagos, patrones de actividad, fobia lunar, viento, Mena Nizanda, Oaxaca.

\begin{abstract}
We analyze the influence of wind speed and the intensity of moonlight in daily activity patterns of 4 species of phyllostomid bats of Mena Nizanda, Juchitán, Oaxaca. When we consider all the data set, We found a significant and inverse correlation between the activity of bats and the wind speed, while the intensity of moonlight showed no statistically significant association with the activity of bats. The higher activity was observed with wind speed from 24 to $15.5 \mathrm{~km} / \mathrm{h}$ and high luminosity (4 on a scale from 0 to 5 ). When we considering only the activity of Choeroniscus godmani, a small-size nectar-feeding species and the most abundant species of the community, activity was correlated positively and significantly with the intensity of moonlight, and inversely and significantly with the wind speed. When we considered data of 3 large-size fruit-eaten specialists in Ficus of the genus Artibeus, we did not find statistically significant correlation with neither wind speed or intensity of moonlight. The above results, as well as the act that the number of captures of $C$. godmani and those of three species of Artibeus do not show significant correlation, support the hypothesis that the activity patterns of bats are affected by the 2 environmental factors studied in a species-specific way.
\end{abstract}

Key words: activity patterns, bats, lunar phobia, wind speed, Mena Nizanda, Oaxaca.

\section{Introducción}

La actividad de los murciélagos se ve influenciada por una serie de factores tanto intrínsecos como extrínsecos. Entre estos últimos están la intensidad de la luz lunar, la temperatura, la precipitación, la velocidad del viento y

Recibido: 02 junio 2009; aceptado: 08 enero 2010 los factores sociales (Erkert, 1982), la disponibilidad de alimento (Korine et al., 2000) y la estructura física del ambiente (Jaberg y Guisan, 2001). Entre los factores ambientales, el más estudiado ha sido la intensidad de la luz lunar. Se ha documentado que varias especies de murciélagos neotropicales responden a ella reduciendo su actividad de vuelo durante las noches claras (Mancina, 2008), lo que se conoce como fobia lunar, y su efecto es variable; por ejemplo, en noches con luz, Carollia perspicillata se des- 
plaza en promedio sólo el $21 \%$ de la distancia que recorre en noches obscuras (Heithaus y Fleming, 1978), mientras que el murciélago vampiro Desmodus rotundus probablemente no abandona sus refugios diurnos en noches de luna llena (Flores-Crespo et al., 1972).

Es probable que tanto la velocidad del viento como la intensidad de la luz lunar afecten sólo algunos aspectos de la actividad diaria. por ejemplo, en Barro Colorado, Panamá, en noches con luna brillante el vuelo de Artibeus jamaicensis y Vampyrodes caraccioli en torno a los árboles con frutos maduros de los que se alimentan se reduce considerablemente e incluso se suspende, pero otros desplazamientos como los de búsqueda y hacia sitios de percha continúan normalmente (Morrison, 1980). Similarmente, D. rotundus (Flores-Crespo et al., 1972) y el murciélago pescador Noctilio leporinus (Börk, 2006) muestran una clara relación entre el forrajeo y la ausencia de luz lunar. Esta disminución de actividad es una estrategia que minimiza el riesgo de depredación, por lo que actividades como la alimentación se prolongan más en noches obscuras (Börk, 2006).

La intensidad de la fobia lunar se ve afectada por varias características, como los sitios de forrajeo (Thies et al., 2006), incluso en especies que comparten hábitos alimentarios; por ejemplo, las especies del género Artibeus son frugívoras que forrajean en el dosel y sus patrones de actividad muestran una relación inversa con el grado de intensidad de la iluminación lunar, mientras que en el mismo sitio, Carollia castanea, otro filostómido frugívoro, pero que forrajea en el sotobosque, no muestra reducción significativa en la actividad de vuelo en las noches brillantes cercanas a la luna llena. Para C. castanea las limitaciones ecológicas (tamaño corporal pequeño asociado con tasa metabólica alta) son los factores más importantes para explicar los patrones de actividad (Thies et al., 2006).

El efecto de otros factores en los patrones de actividad diaria de los murciélagos es menos conocido. Uno de ellos es el viento, que cuando aumenta su velocidad disminuye la capacidad de detección de las presas, especialmente aquella de especies que se alimentan de insectos, afectando principalmente a quienes emiten frecuencias constantes (Ayuso-Oliva, 2005). Por esta razón los murciélagos insectívoros prefieren cazar en zonas menos expuestas al viento, donde además la densidad de presas es mayor (Lewis y Stephenson, 1996; Boonman, 1999). Otros estudios revelan que algunas especies de murciélagos evitan cazar sobre cuerpos de agua turbulenta, donde el viento incrementa las ondas en la superficie de las masas de agua creando ecos confusos que afectan la ecolocalización (Boonman et al., 1998; Rydell et al., 1999).

A pesar de su importancia, en México existen muy poco estudios sobre la relación entre factores ambientales y la actividad de murciélagos, por lo que el objetivo de esta investigación es conocer la relación entre los patrones de actividad de murciélagos filostómidos, la luminosidad de la luna y la intensidad del viento, así como su efecto en distintos gremios tróficos en la zona de Mena Nizanda, en el estado de Oaxaca, en el sureste de México.

\section{Materiales y métodos}

El estudio se realizó en el ejido Mena Nizanda $\left(16^{\circ} 38^{\prime} 35.16^{\prime \prime} \mathrm{N}, 95^{\circ} 00^{\prime} 34.53^{\prime \prime} \mathrm{O}\right)$, en el municipio de Asunción Ixtaltepec, distrito de Juchitán, en la región del istmo de Tehuantepec, en el estado de Oaxaca. La región está bordeada al oeste y suroeste por la sierra Madre del Sur (sierra de Miahuatlán), al noroeste por la sierra Norte de Oaxaca (Sierra Mixe), al este por la sierra Tolistoque, y al sur por los terrenos llanos de la planicie costera del golfo de Tehuantepec y cubre aproximadamente $85 \mathrm{~km}^{2}$. Mena Nizanda se localiza en una zona conocida como Paso de Chivela, localizada en los límites de la región central y la de la planicie costera de Tehuantepec. En ella es notoria la transición entre el clima cálido húmedo del golfo de México al tropical estacional característico de la vertiente mexicana del Pacífico, una de las áreas menos estudiadas de la vertiente pacífica del Istmo, pero posiblemente una de las más interesantes biológicamente (Pérez-García et al., 2001). Los vientos alisios, también conocidos como nortes, son muy comunes en esta región y otras zonas del istmo de Tehuantepec. Cuando se presentan, su duración varía de 3 a 5 días, y aunque ocurren todo el año, son más frecuentes, con intervalos de 10 a 15 días, y más intensos de noviembre a abril; esto se debe a que los vientos alisios provenientes del golfo de México se encuentran en las serranías que circundan las partes bajas del istmo de Tehuantepec (Gallardo-Cruz et al., 2005).

En Mena Nizanda se reconocen 7 tipos de vegetación, con predominio de selva baja caducifolia, selva mediana subperennifolia, bosque de galería y vegetación secundaria (Pérez-García et al., 2001). La temperatura media anual es de $25^{\circ} \mathrm{C}$, la temporada seca va de noviembre a abril y la lluviosa de mayo a octubre (García, 1988; Pérez-García et al., 2001; Gallardo-Cruz et al., 2005).

La captura de los ejemplares se realizó con 3 redes de niebla de $6 \times 2.5 \mathrm{~m}$ que se colocaron al atardecer y se retiraron al amanecer o en ausencia total de actividad de murciélagos (i. e., ninguna captura a lo largo de 2 horas consecutivas). En cada sesión de muestreo, las redes se colocaron en lugares distintos para evitar que los ejemplares pudieran reconocer los sitios y evitar las redes (Larsen 
et al., 2007). A cada individuo capturado se le colocó en el cuello una banda sujeta-cables de plástico marca Steren, modelo TY300 de $100 \mathrm{~mm}$ de largo por 2.5 de ancho y 1 $\mathrm{g}$ de peso, las cuales incluyen un número de serie grabado.

La velocidad del viento se estimó por medio de la escala de Beaufort (Comisión Nacional del Agua, 2001). Aunque es una de las primeras escalas para estimar la velocidad del viento y sus efectos a partir de evidencias visuales, esta escala, propuesta en 1805, sigue siendo usada. Toma valores de cero (calma total, sin vientos o con una velocidad máxima de $1 \mathrm{~km} / \mathrm{h}$ ) a 12 (huracán, con velocidad del viento de $118 \mathrm{~km} / \mathrm{h}$ o mayor). La intensidad de la luminosidad lunar se midió con la escala propuesta por Börk (2006), modificada para incluir los criterios de condición del cielo de la Comisión Nacional del Agua (2001). La escala toma valores de 0 a 5 , con los valores más altos correspondiendo a mayor luminosidad, que se presenta por la combinación de cielo despejado y luna llena. Tanto la intensidad del viento como la luminosidad se estimaron al principio y al final de cada sesión de muestreo, y el promedio de ambos valores se utilizó en el análisis. La actividad de los murciélagos se expresó por medio del número total de ejemplares capturados por noche. La relación entre la intensidad del viento, luminosidad de la luna y actividad de los murciélagos se analizó con el coeficiente de correlación no paramétrico de Spearman $(\rho)$ (Hollander y Wolfe, 1999), tanto para toda la comunidad, como para cada uno de los gremios tróficos representados y clases de tamaño presentes en ella, de acuerdo con Medellín (1993).

\section{Resultados}

El estudio se realizó de agosto de 2007 a febrero de 2008, con un esfuerzo de colecta de 1638 metros red hora desplegado durante 20 noches, con el que se capturaron 117 ejemplares, lo que representó un éxito de captura de 0.07 murciélagos/red·hora o 5.85 murciélagos/noche. Se registraron 6 especies de murciélagos filostómidos: Choeroniscus godmani (77 individuos), Glossophaga soricina (1), Artibeus intermedius (24), A. jamaicensis (11), A. lituratus (3) y Carollia brevicauda (2). Estas especies representan 3 gremios tróficos: nectarívoros las 2 primeras, frugívoros especialistas en Ficus las 3 especies de Artibeus (Giannini y Kalko, 2004; Thies et al., 2006) y C. brevicauda es frugívora especialista en Piper y Cecropia (Thies et al., 2006). Para el análisis de la relación entre la velocidad del viento, la intensidad de la iluminación lunar y la actividad no se incluyó el último gremio porque el tamaño de muestra fue muy pequeño, y en el caso de los nectarívoros sólo se consideraron los datos de C. godmani porque pertenece a una clase de tamaño más pequeño que G. soricina (Medellín, 1993). Por otra parte, y a diferencia de C. godmani, las especies del género Glossophaga muestran una menor especialización morfológica craneal a la nectarivoría (Tschapka, 2004). G. soricina incluye en su dieta insectos, frutos, polen, néctar, y fragmentos de flores (Gardner, 1977), incluso G. commissarisi cambia de dieta nectarívora a una más frugívora durante los periodos del año en que disminuye la disponibilidad de néctar (Tschapka, 2004). Esto tiene relevancia porque las especies de glosofaginos que incluyen una mayor proporción de insectos en su dieta muestran mayor eficiencia en su ecolocalización que aquellas que interactúan exclusivamente con plantas quiropterófilas (Howell, 1974), mientras que aquellas que se especializan en la depredación de frutos los localizan principalmente por medio del olfato (Thies et al., 1998), lo que permite inferir que existe un efecto diferente de la velocidad del viento en estas especies.

Para las 4 especies en conjunto los valores más altos de actividad se observaron consistentemente con luminosidad alta (4), que se presenta con luna llena y cielo medio nublado o luna en cuarto menguante y/o creciente con cielo despejado. Bajo estas condiciones se capturaron en promedio 7.83 individuos por noche (desviación estándar 7.9), y en 4 de las 12 noches en que se observó esta condición se obtuvieron números altos de capturas, con más de 10 individuos en cada una de las noches, mientras que en ninguna de las 8 en que se presentaron los valores extremos de luminosidad se capturó más de 10 ejemplares. Cuando se presentó la menor luminosidad (1 noche), que resulta de la combinación de luna nueva y cielo medio nublado, el promedio de capturas fue de 1.5 ejemplares (desviación estándar 1.73), y en aquellas noches con la mayor luminosidad (5), caracterizadas por luna llena y cielo despejado, el promedio fue de 4.25 (desviación estándar 3.68). El coeficiente de correlación no paramétrico de Spearman muestra que la actividad de los murciélagos no está relacionada significativamente con la luminosidad de la luna $(\rho=0.186, P=0.43)$.

Las noches con capturas numerosas presentaron vientos de intensidad de 3.5 a 4.5 (15.5 a $24 \mathrm{~km} / \mathrm{h})$, mientras que la actividad fue nula a partir de que el viento alcanzó intensidad 6 ( 29 a $38 \mathrm{~km} / \mathrm{h}$ ). La intensidad del viento está correlacionada en forma inversamente proporcional y significativa con la actividad de murciélagos $(\rho=-0.473, P=$ 0.035). La intensidad del viento y la luminosidad fueron variables estadísticamente independientes $(\rho=0.402, P=$ 0.079).

El número de capturas de ambos grupos (C. godmani por una parte y las 3 especies de Artibeus conformando el segundo) no mostró correlación estadísticamente significativa $(\rho=0.0872, \mathrm{P}=0.7144)$. Para $C$. godmani la actividad 

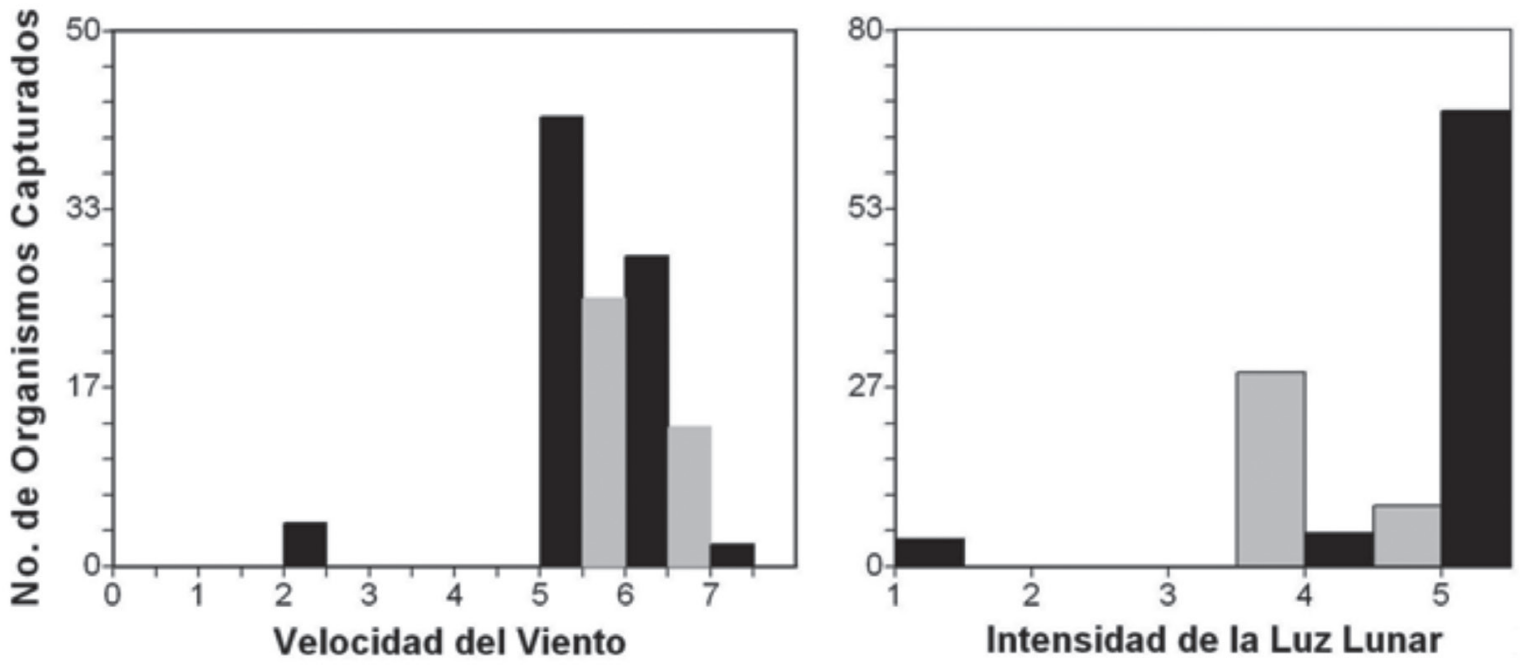

Figura 1. Frecuencia de organismos capturados con distintas velocidades del viento e intensidad de la luz lunar en Mena Nizanda. Las barras de color claro representan las frecuencias para tres especies del género Artibeus y las obscuras las de Choeroniscus godmani.

estuvo correlacionada en forma positiva y significativa con la intensidad de la luz lunar $(\rho=0.574, \mathrm{P}=0.008)$ y la velocidad del viento mostró una relación significativa pero inversamente proporcional con la actividad $(\rho=-0.532, \mathrm{P}=$ 0.015). Para las especies de Artibeus la actividad no mostró relación significativa con la intensidad de la luz lunar ( $\rho=0.1289, P=0.588)$, ni con la velocidad del viento $(\rho=$ $0.331, \mathrm{P}=0.153$; Fig. 1).

\section{Discusión}

Las 4 especies de murciélagos estudiadas en Mena Nizanda muestran actividad a velocidades del viento más altas que las registradas en otras zonas, pues la mayor actividad ocurre entre los 15.5 y los $24 \mathrm{~km} / \mathrm{h}$ y cesa cuando alcanza los $29 \mathrm{~km} / \mathrm{h}$, mientras que por ejemplo en Nueva York, la actividad de murciélagos insectívoros (familia Vespertilionidae) cesó totalmente cuando alcanzó velocidades mínimas de $12.24 \mathrm{~km} / \mathrm{h}$, y los días sin actividad se caracterizaron por velocidades mínimas de 7.16 a 17.24 $\mathrm{km} / \mathrm{h}$ (Reynolds, 2006).

Otros estudios sobre el efecto de la intensidad de la luz lunar en la actividad de comunidades de murciélagos en zonas tropicales y subtropicales muestran que no todas las especies responden de la misma forma a este factor. Por ejemplo, para una comunidad de murciélagos filostómidos en el sureste de Brasil, el mayor número de capturas se registró cuando la luna presentó iluminación del 26-50\% de su cara, es decir, fobia lunar, pero de las 10 especies más abundantes, sólo 4 mostraron esta respuesta en forma estadísticamente significativa, mientras que las otras 6 no mostraron ninguna relación significativa (Esbérard, 2007).

Al considerar en conjunto los datos de las 4 especies de murciélagos más abundantes en Mena Nizanda, los resultados no apoyan la hipótesis de que la actividad está determinada por la intensidad de la luz lunar; en cambio, indica que la velocidad del viento es más importante. Sin embargo, en este conjunto de especies, a diferencia de otros de ambientes tropicales o subtropicales, la especie dominante fue Choeroniscus godmani $(65.8 \%$ de los ejemplares capturados), que es de hábitos nectarívoros y se considera como localmente rara en los sitios en que habita (Arita, 1993; Olguín-Monroy et al., 2008). Con excepción del caso de Mena Nizanda y una comunidad en Costa Rica (Tschapka, 2004), se desconoce la existencia de otras comunidades de murciélagos en que predominen especies con estos hábitos alimentarios.

La dieta nectarívora representa un reto fisiológico importante, porque el recambio energético es considerablemente mayor que para especies insectívoras o hematófagas, debido a que el néctar está compuesto por $82.6 \%$ de agua, $17 \%$ de azúcar, hasta $4 \%$ de proteínas y $0 \%$ de grasa (Neuweiler, 2000; Tschapka, 2004). Esto, junto con tasas metabólicas más altas y por lo tanto requerimientos energéticos proporcionalmente altos de individuos de especies de talla pequeña, como C. godmani, podrían explicar que 
la luminosidad sea un factor secundario en la determinación de los patrones de actividad de la comunidad en su conjunto. Además, la velocidad del viento afecta más a los murciélagos de talla pequeña (Verboom y Huitema, 1997).

La ausencia de correlación entre el número de capturas de los 2 grupos de especies estudiados puede ser una evidencia de que la actividad se ve afectada en forma distinta por los factores ambientales en cada conjunto. La correlación significativa de la actividad y la luminosidad de la luna en $C$. godmani es contrario a lo observado en 2 especies nectarívoras del género Lonchophylla en una comunidad en el sureste de Brasil, que se capturaron sólo cuando se presentó la fase más oscura de la luna (Esbérard, 2007), aunque a diferencia de C. godmani en Mena Nizanda, la abundancia de esas especies fue muy baja y no permitió análisis estadístico.

La falta de correlación entre la actividad, la velocidad del viento y la intensidad de la luz lunar para las especies de Artibeus en Mena Nizanda es opuesta a lo observado en otros sitios, donde al menos A. jamaicensis y A. lituratus mostraron fobia lunar (Esbérard, 2007; Erkert, 1974). Además, dado que la localización de los frutos por parte de los murciélagos depende en parte de su olor (Korine et al., 2000), se esperaría que el viento tuviera una relación significativa con la actividad de los miembros de este gremio, sobretodo en una zona como Mena Nizanda, caracterizada por la presencia regular de corrientes fuertes. Esta misma situación de patrones de actividad diferentes para la misma especie se ha observado en otras especies como Phyllostomus astatus, que presenta fobia lunar en unos sitios (Carranza-Almanza y Arias-de-Reyna Martínez, 1982) mientras que en otros no muestra ninguna relación (Erkert, 1976). Para Noctilio leporinus se ha observado algo similar: actividad constante independiente del grado de iluminación de la luna (Brown, 1968) o movimientos restringidos al crepúsculo y al amanecer (Carranza-Almanza y Arias-de-Reyna Martínez, 1982). Los ritmos de actividad de una especie pueden diferir en gran medida dependiendo de la proximidad de los sitios de refugio diurno (Fenton y Kunz, 1977).

Otros factores no evaluados que podrían ser determinantes en la actividad de los murciélagos de Mena Nizanda son la disponibilidad de alimento (Hayes, 1997; Lang et al., 2006) y su variación a través del tiempo (Hayes, 1997; Chávez y Ceballos, 2001). Estudios de radiotelemetría en A. jamaicensis indican que las distancias que recorre diariamente esta especie están relacionadas con la densidad y distribución de árboles con frutos maduros (Morrison, 1978). Por otra parte, los depredadores pueden representar una importante fuerza selectiva que afecta los patrones de actividad diaria (Karlsson et al., 2002). Por ejemplo, en Panamá, durante los periodos con luna brillante, $A$. lituratus suspende o reduce notoriamente sus viajes para alimentación a árboles con frutos, aunque otros movimientos continúan incluso en noches de luna llena. De la misma forma, en especies de hábitos insectívoros del género Pipistrellus, la evasión de depredadores es el factor más importante en la determinación del patrón de movimientos, aun con niveles relativamente altos de iluminación lunar (Verboom y Spoelstra, 1999). Finalmente, también cabe la posibilidad de que los individuos sigan moviéndose durante los periodos de mayor iluminación de la luna, pero en sitios o microhábitats diferentes a aquellos que visitan cuando hay oscuridad total (Hecker y Brigham, 1999).

\section{Agradecimientos}

El Instituto Politécnico Nacional proporcionó apoyo económico para la realización del proyecto (apoyos SIP-20070826 y SIP-20080431 a A. Santos-Moreno). Agradecemos a las autoridades de Mena Nizanda las facilidades y la autorización para la ejecución del trabajo de campo. A. Alfaro Espinosa y J. L. García-García, G. Pérez Irineo y G. González Pérez revisaron versiones preliminares del documento. L. Léon Paniagua y un revisor anónimo aportaron importantes ideas que mejoraron significativamente la calidad del manuscrito.

\section{Literatura citada}

Arita, H. 1993. Rarity in Neotropical bats: correlations with phylogeny, diet, and body mass. Ecological Applications 3:506-517.

Ayuso-Oliva, A. M. 2005. Uso del hábitat en quirópteros en un área alterada por la construcción por la línea de tren de alta velocidad en el sur de la península Ibérica. Implicaciones para su conservación y estudio de los registros sonoros del genero Pipistrellus. Tesis, doctorado Facultad de Ciencias, Universidad de Granada. 251 p.

Boonman, A. M., M. Boonman., F. Bretschneider y W.A. Van de Grind. 1998. Prey detection in trawling insectivorous bats: duckweed affects hunting behaviour in Daubenton's bat Myotis daubentonii. Behavioural Ecology and Sociobiology 44:99-107.

Boonman, M. 1999. Monitoring bats on their hunting grounds. Myotis 34:99-107.

Börk, K. S. 2006. Lunar phobia in the greater fishing bat (Noctilio leporinus) (Chiroptera: Noctilionidae). Revista de Biología Tropical 54:1117-1123.

Brown, J. H. 1968. Activity patterns of some neotropical bats, Journal of Mammalogy 49:754-757. 
Carranza-Almanza, J. y L. Arias-de-Reyna Martínez. 1982. Ritmos de actividad de cuatro especies de murciélagos neotropicales. Historia Natural 2:213-220.

Chávez, C. y G. Ceballos. 2001. Diversidad y abundancia de murciélagos en selvas secas de estacionalidad contrastante en el oeste de México. Revista Mexicana de Mastozoología 5:27-44.

Comisión Nacional del Agua. 2001. Prácticas operativas de mantenimiento en estaciones climatológicas convencionales. Manual para el personal operador (gratificado). Redes climatológicas. Informe final No. 67-02. Organización Meteorológica Mundial-Coordinación del Programa de Modernización del Manejo del Agua-Gerencia de Aguas Superficiales e Ingeniería de Ríos, México, D.F. 16 p.

Erkert, H. G. 1974. The effect of moonlight on the activity of nocturnal mammals. Oecologia 14:269-287.

Erkert, H. G. 1976. Lunarperiodic variation of the phase-angle difference in nocturnal animals under natural Zeitgeberconditions near the equator. Internatinal Journal of Chronobioogy 4:125-38.

Erkert, H. G. 1982. Ecological aspects of bat activity rhythms. In The ecology of bats, T. H. Kunz (ed.) Plenum, New York. p. 201-242.

Esbérard, C. E. L. 2007. Influence of moon cycle in phyllostomid bat capture. Iheringia. Série Zoologia 97:81-85.

Fenton, M. B. y T. H. Kunz. 1977. Movements and behavior. In Biology of bats of the New World family Phyllostomatidae, part II, R. J. Baker, J. K. Jones Jr. y D. C. Carter (eds.). Special Publications, of the Museum of Texas Tech University 13. Texas Tech University, Lubbock. p. 351-364.

Flores-Crespo, R., S. B. Linhart, R. J. Burns y G. C. Mitchell. 1972. Foraging behavior of the common vampire bat related to moonlight. Journal of Mammalogy 53:366-368.

Gallardo-Cruz, J. A., J. A. Meave y E. Pérez-García. 2005. Estructura, composición y diversidad de la selva baja caducifolia del Cerro Verde, Nizanda (Oaxaca), México. Boletín de la Sociedad Botánica de México 76:19-35.

García, E. 1988. Modificaciones al sistema de Köppen (para adaptarlo a las condiciones de la Republica Mexicana), cuarta edición.. Editado por la autora, México, D.F.

Gardner, A. L. 1977. Feeding habits. In Biology of bats of the New World family Phyllostomatidae, part II, R. J. Baker, J. K. Jones, Jr. y D. C. Carter (eds.). Special Publications of the Museum of Texas Tech University 13. Texas Tech University, Lubbock. p. 293-350.

Giannini, N. P. y E. K. V. Kalko. 2004. Trophic structure in a large assemblage of phyllostomid bats in Panama. Oikos 105:209-220.

Hayes, J. P. 1997. Temporal variation in activity of bats and the design of echolocation-monitoring studies. Journal of Mammalogy 78:514-524.

Hecker, K. R. y R. M. Brigham. 1999. Does moonlight change vertical stratification of activity by forest-dwelling insectivorous bats? Journal of Mammalogy 80:1196-1201.

Heithaus, E. R y T. H. Fleming. 1978. Foraging movements of a frugivorous bat, Carollia perspicillata (Phyllostomatidae). Ecological Monographs 48:127-143.

Hollander, M. y D. A. Wolfe. 1999. Nonparametric statistical methods, segunda edición, Wiley Series in Probability and Statistics, Wiley, Hoboken, New Jersey. 787 p.

Howell, D. J. 1974. Acoustic behavior and feeding in Glossophagine bats. Journal of Mammalogy 55:293-308.

Jaberg, C. y A. Guisan. 2001. Modelling the distribution of bats in relation to landscape structure in a temperate mountain environment. Journal of Applied Ecology 38:1169-1181.

Karlsson, B. L., J. Ekllof y J. Rydell. 2002. No lunar phobia in swarming insectivorous bats (family Vespertilionidae). Journal of Zoology 256:473-477.

Korine, C., E. K.V. Kalko y E. A. Herre. 2000. Fruit characteristics and factors affecting fruit removal in a Panamanian community of strangler figs. Oecologia 123:560-568.

Lang, A. B., E. K. V. Kalko, H. Römer, C. Bockholdt y D. K. N. Dechmann. 2006. Activity levels of bats and katydids in relation to the lunar cycle. Oecologia 146:659-666.

Larsen, R. J., K. A. Boegler, H. H. Genoways, W. P. Masefield, R. A. Kirsch y S. C. Pedersen. 2007. Mist netting bias, species accumulation curves, and the rediscovery of two bats on Montserrat (Lesser Antilles). Acta Chiropterologica 9:423435 .

Lewis, T. y J. W. Stephenson. 1996. The permeability of artificial windbreaks and the distribution of flying insects in the leeward sheltered zone. Annals of Applied Biology 60:355363.

Mancina, C. 2008. Effect of moonlight on nocturnal activity of two Cuban nectarivores: the Greater Antillean Longtongued Bat (Monophyllus redmani) and Poey's Flower Bat (Phyllonycteris poeyi). Bat Research News 49:71-74.

Medellín, R. A. 1993. Estructura y diversidad de una comunidad de murciélagos en el trópico húmedo mexicano. In Avances en el estudio de los mamíferos de México, R. A. Medellín y G. Ceballos (eds.). Asociación Mexicana de Mastozoología, México, D.F. p. 333-354.

Morrison, D. W. 1978. Foraging ecology and energetics of the frugivorous bat Artibeus jamaicensis. Ecology 59:716-723.

Morrison, D. W. 1980. Foraging and day-roosting dynamics of canopy fruit bats in Panama. Journal of Mammalogy 61:2029.

Neuweiler, G. 2000. The biology of bats. Oxford University Press, New York. 310 p.

Olguín-Monroy, H., L. León-Paniagua, U. Melo Samper-Palacios y V. Sánchez-Cordero. 2008. Mastofauna de la región de Los Chimalapas, Oaxaca, México. In Avances en el estudio de los mamíferos de México II, E. Espinoza Medinilla, C. Lorenzo Monterrubio y J. Ortega (eds.). Publicaciones especiales, vol. 
II, Asociación Mexicana de Mastozoología, México, D. F. p. 165-216.

Pérez-García, E. A., J. Meave y C. Gallardo. 2001. Vegetación y flora de la región de Nizanda, Istmo de Tehuantepec, Oaxaca, México. Acta Botanica Mexicana 56:19-88.

Reynolds, S. D. 2006. Monitoring the potential impact of a wind development site on bats in the Northeast. Journal of Wildlife Management 70:1219-227.

Rydell, J., L. A. Miller y M. E. Jensen. 1999. Echolocation constrains of Daubenton's bat foraging over water. Functional Ecology 13:247-255.

Thies, W., E. K. V. Kalko y H. Schnitzler. 2006. Influence of environment and resource availability on activity patterns of Carollia castanea (Phyllostomidae) in Panama. Journal of Mammalogy 87:331-338.
Thies, W., E. K.V. Kalko y H. Schnitzler. 1998. The roles of echolocation and olfaction in two Neotropical fruit-eating bats, Carollia perspicillata and C. castanea, feeding on Piper. Behav Ecol Sociobiol 42:397-409.

Tschapka, M. 2004. Energy density patterns of nectar resources permit coexistence within a guild of Neotropical flowervisiting bats. Journal of Zoology 263:7-21.

Verboom, B. y H. Huitema. 1997. The importance of linear landscape elements for the pipistrelle Pipistrellus pipistrellus and the serotine bat Eptesicus serotinus. Landscape Ecology 12:117-125.

Verboom, B. y K. Spoelstra. 1999. Effects of food abundance and wind on the use of tree lines by an insectivorous bat, Pipistrellus pipistrellus. Canadian Journal of Zoology 77:1393-1401. 
